University of Nebraska - Lincoln

DigitalCommons@University of Nebraska - Lincoln

\title{
Perceived importance of AAC messages to support communication in rehabilitation settings
}

\author{
Susan Koch Fager \\ Judith M. Burnfield \\ Chase M Pfeifer \\ Tabatha Sorenson
}

Follow this and additional works at: https://digitalcommons.unl.edu/mechengfacpub

Part of the Mechanics of Materials Commons, Nanoscience and Nanotechnology Commons, Other Engineering Science and Materials Commons, and the Other Mechanical Engineering Commons

This Article is brought to you for free and open access by the Mechanical \& Materials Engineering, Department of at DigitalCommons@University of Nebraska - Lincoln. It has been accepted for inclusion in Mechanical \& Materials Engineering Faculty Publications by an authorized administrator of DigitalCommons@University of Nebraska Lincoln. 


\title{
Perceived importance of AAC messages to support communication in rehabilitation settings
}

\author{
Susan Koch Fager, Judith M. Burnfield, \\ Chase M. Pfeifer and Tabatha Sorenson
}

Institute for Rehabilitation Science and Engineering,

Madonna Rehabilitation Hospitals, Lincoln, NE, USA

Correspondence — Susan Koch Fager, sfager@madonna.org ; Institute for Rehabilitation Science and Engineering, Madonna Rehabilitation Hospitals, 5401 South Street, Lincoln, NE 68506, USA

\begin{abstract}
Purpose: Permanent or temporary speech loss can occur due to a variety of medical conditions and often requires individuals to use augmentative and alternative communication (AAC) strategies and technologies to support communication. The use of AAC in medical and rehabilitation settings is critical to ensure the health, safety and psychological well-being of communicatively vulnerable individuals.

Method: This study surveyed the perceived importance of communication messages within five categories (Basic Needs, Patient-Provider Specific Communication, Social, Feelings, and Messages for Young Children) by individuals with disability who have undergone recent medical care as well as by rehabilitation care providers.

Results: Results indicated that, with only a few exceptions, participants with disability more frequently selected all of the messages listed under the Patient-Provider Specific, Social, and Feelings categories compared to rehabilitation care providers' responses. Additionally, the individuals with disabilities selected messages that were more personalized to their specific care needs under Basic Needs.
\end{abstract}

Published in Disability and Rehabilitation: Assistive Technology 16:7 (2021), pp 796-801. doi:10.1080/17483107.2020.1736652

Copyright (c) 2020 Informa UK Limited, trading as Taylor \& Francis Group. Used by permission. Submitted 26 November 2019; accepted 26 February 2020. 
Both participant groups were asked to also suggest other messages that they perceived as important under each category. The other suggested messages demonstrated the potential need to provide personalization to AAC displays to best meet the communication needs of individuals with disability.

Implications for rehabilitation: There is a critical need for individuals who find themselves in medical settings (ICU, acute care hospital, rehabilitation hospital, etc.) to be able to communicate. This study provides preliminary information on the breadth of communication content that is perceived as relevant by individuals with disability and rehabilitation care providers. The need to provide personalized as well as a wide range of communication content options was of particular importance to individuals with disability. This information may provide specific guidance on how to best develop augmentative and alternative communication options for individuals in medical settings.

Keywords: Augmentative and alternative communication, rehabilitation, patientprovider communication, disability, assistive technology

\section{Introduction/background}

C.J. was a 30 year-old male with sudden onset Guillain-Barré which rapidly left him paralyzed, on a ventilator, and unable to speak. He had to rely on hospital staff to ask him yes/no questions and used eye movements to respond. C.J. eventually recovered his ability to speak, but reflected on his experiences of being unable to fully express all the thoughts going through his mind early in his acute care hospitalization and during his transition to rehabilitation. He had many questions about what was happening, his prognosis, and treatments that might help his condition. He had many emotions he wished to express and wanted to be able to communicate these to his wife and children. However, his only method of communication relied on his communication partner's ability to guess what he might want to talk about using yes/no questions. This severely limited C.J's ability to communicate during this medically critical and stressful time during his recovery.

There is a critical need for individuals who find themselves in medical settings (ICU, acute care hospital, rehabilitation hospital, etc.) to be able to communicate. Hospital adverse events often occur due to communication breakdowns [1], and individuals with communication impairments are three times more likely to experience adverse events when hospitalized [2]. Regardless of whether communication impairments are temporary (e.g., oral intubation, temporary paralysis) or 
permanent (e.g., following a neurologic injury or illness), the inability to communicate while hospitalized can have lasting psychological effects on those who are communicatively vulnerable [3].

Despite barriers, communicatively vulnerable individuals have a fundamental right to engage in patient-provider communications (PPC) in medical settings and engage actively in their care [3]. The Joint Commission [4] adopted the following definition of PPC: "Effective communication is the successful joint establishment of meaning wherein patients and providers exchange information, enabling patients to participate actively in their care from admission through discharge, and ensuring the responsibilities of both patients and providers are understood. To be truly effective, communication requires a two-way process between patient and provider in which messages are negotiated until information is correctly understood by both parties. Successful communication takes place only when providers understand and integrate the information gleaned from patients, and when patients comprehend accurate, timely, complete, and unambiguous messages from providers in a way that enables them to participate responsibly in their care." $[4, p .1]$.

Communication impairments often necessitate use of augmentative and alternative communication (AAC) strategies and technologies to support expression, but use is inconsistent. Communication supports using AAC can range from "low tech" (e.g., gestures, writing, paper communication boards) to "high tech" (e.g., tablet and computerbased devices with software and apps that include pictures, symbols, and onscreen keyboards for spelling messages) [5]. Staff expertise can drive AAC selection [6]. For example, many intensive care units (ICUs) and acute care settings may lack AAC knowledgeable therapists (e.g., speech-language pathologists), [7-9] and thus rely on nurses to identify and implement AAC strategies with communicatively vulnerable individuals. In the ICU, nurses frequently employ gestures/expressions, answering yes/no questions, and handwriting to communicate content that frequently focuses on basic needs such as pain $[6,10]$.

Beyond basic needs, patients and families have also expressed a desire to communicate more individualized, personalized, and emotional content in ICU and acute hospital settings as well as engage in conversations regarding end-of-life decisions [10-12]. As individuals transition to rehabilitation hospitals, their need to communicate on a 
wide range of topics to actively engage in their care and connect with their friends and family continues to expand [13].

Supporting the needs of communicatively vulnerable individuals in medical settings requires not only the appropriate AAC technology, but also knowledge, skills and staff expertise. While a wide range of communication boards and software/applications have been designed to support communication in medical settings, there is paucity of evidence in the literature as to the specific messages that might be most beneficial to include in AAC systems (low or high tech) to support communication in medical settings. This preliminary study investigated the importance of communication content options for individuals with communication impairment receiving inpatient care and for care providers interacting with those patients in a rehabilitation setting. This work represents a critical first step to advancing understanding of the content needs of patients and the diverse professional team with whom they communicate.

\section{Method}

\section{Participants}

The participants included 10 individuals with disabilities who were currently receiving care at a free-standing rehabilitation hospital in the Midwest. Additionally, 128 rehabilitation providers who worked in the rehabilitation hospital participated. The individuals with disability were referred by their primary therapist or a nurse familiar with them. Rehabilitation care providers were recruited via an email survey.

\section{Individuals with disabilities}

This group consisted of nine males and one female who had the following diagnoses and received care for this diagnosis or complications related to the diagnosis within the past 5 years: Guillain-Barré syndrome (2), spinal cord injury (5), cerebral palsy (1), brainstem stroke (1), and traumatic brain injury (1). Two participants had been unable to communicate early in their recovery and relied on AAC to support communication (switch scanning and eye tracking devices as 
well as low tech alphabet boards). Both used power wheelchairs (one with head control and one with joystick control) to negotiate their environments. Eight individuals used their natural speech to support communication during their recovery. Of these individuals, six used power wheelchairs with either joystick or head control, and two had progressed to independent mobility without a wheelchair by the time they completed the survey.

\section{Rehabilitation providers}

This group was comprised of 6 case managers/social workers, 3 neuropsychologists, 34 nurses, 30 occupational therapists, 2 physiatrists, 22 physical therapists, 3 recreational therapists, 3 respiratory therapists, and 25 speech-language pathologists who collectively averaged 10 years working in their current discipline (range of $<1-35$ years). Of these participants, $12 \%$ worked only with pediatric patients, $68 \%$ worked only with adult/geriatric patients, and $20 \%$ worked with both pediatric and adult/geriatric patients. The diagnoses providers reported serving included stroke (64\%), traumatic brain injury (63\%), spinal cord injury (38\%), patients dependent on ventilator support (26\%), orthopedic injury (22\%), degenerative diseases (e.g., amyotrophic lateral sclerosis, multiple sclerosis) (20\%), developmental disabilities (e.g., cerebral palsy, Down Syndrome) (11\%), burns (9\%), and other neurologic disabilities and diagnoses (e.g., Guillain-Barré syndrome, medically complex, cardiac rehabilitation, dementia, wound) (7\%).

\section{Materials}

A printed survey was prepared for patient participants and a similar online version of the survey was prepared for rehabilitation providers. The survey included categories of messages commonly used on communication boards and in communication device software. Content was divided into five key categories: Basic Needs, Patient-Provider Specific Communication, Social, Feelings, and Messages for Young Children. Ten to 15 example messages were given under each category. Space was provided following each category for respondents to include other messages that may not have been reflected in the examples given. 


\section{Procedures}

Following IRB approval of the study, potential participants were invited to complete a paper version of the survey (individuals with physical disabilities) or an online survey (rehabilitation care providers). The paper survey for individuals with disabilities requested basic demographic information including gender, diagnosis, and history of using AAC. A research team member was available to assist individuals with physical disabilities in completing the survey (i.e., physically recording participant's desired responses). The rehabilitation care providers' electronic survey collected background/demographic information including discipline and years of experience working with select patient populations. The survey included the following instructions for both groups: "This survey will provide you with examples of messages that patients might want to communicate. You will be asked to indicate what messages would be most appropriate and to provide additional message ideas that may not have been included."

\section{Data analysis}

Data were analyzed descriptively. The percentage of respondents that selected a message was calculated. Written responses related to recommendations for additional messages to include were analyzed to identify common themes and for frequency (e.g., how many respondents indicated a specific additional message needed to be included). When two or more participants recommended similar content, then this content was identified as a theme.

\section{Results}

\section{Messages selected as important for a patient to be able to com- municate in a medical setting}

The percentage of individuals with a disability and the percentage of rehabilitation care providers indicating that specific messages were important for patients to be able to communicate in a medical setting are displayed in red and grey, respectively, within the categories of 


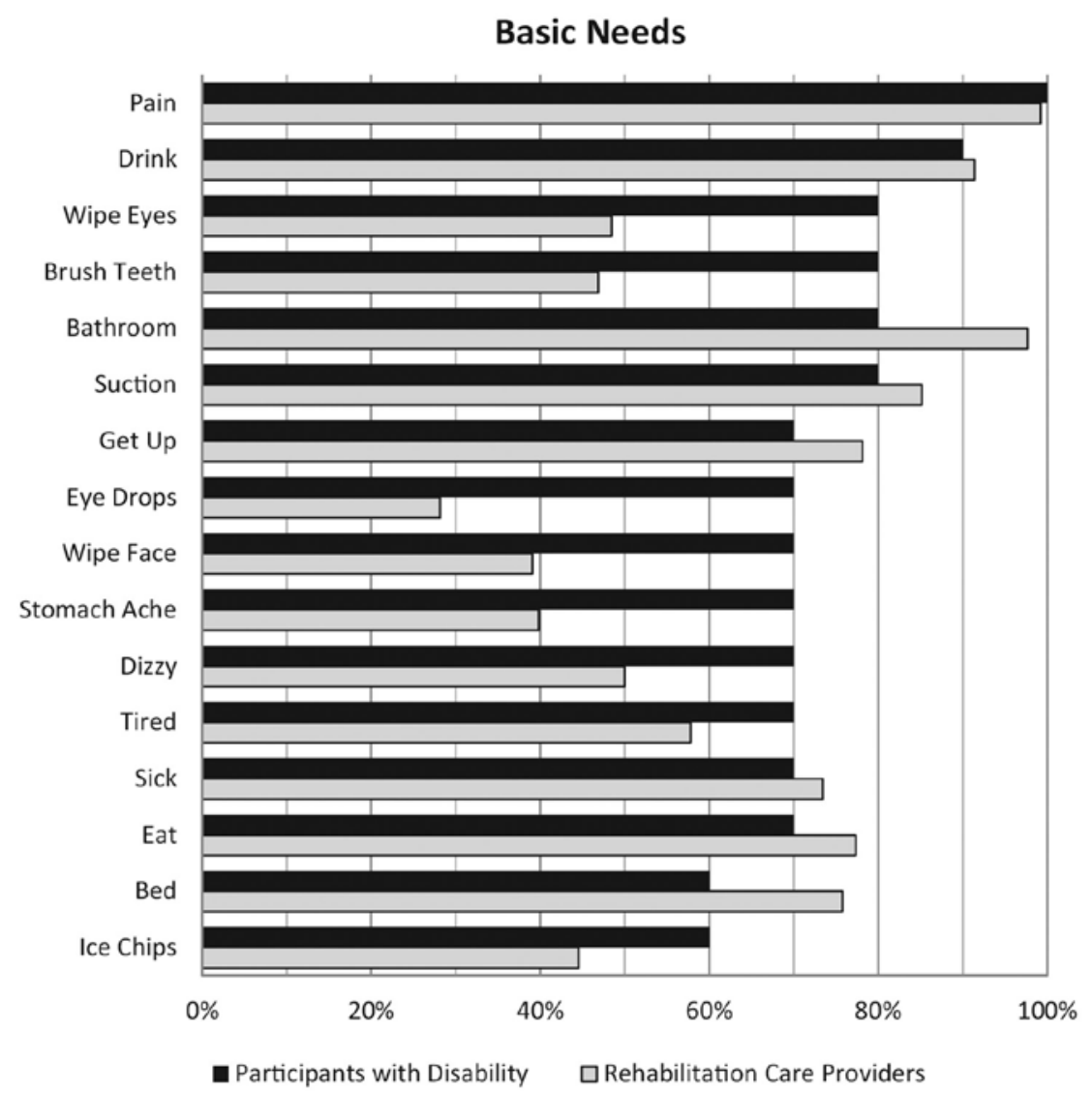

Figure 1. Percentage of Basic Needs messages selected as important by individuals with disability and rehabilitation care providers.

Basic Needs (Figure 1), Patient-Provider (Figure 2), Social (Figure 3), and Feelings (Figure 4). The percentage of rehabilitation care providers indicating that a specific message was important to be able to communicate in a medical setting for pediatric patients is provided in Figure 5. This survey did not include children, thus no data from children are included on Figure 5.

\section{Other suggested messages}

Participants with disabilities and rehabilitation care providers identified additional messages they thought were missing but important to include within each of the categories including Basic Needs $(\mathrm{n}=87$ additional messages suggested), Patient-Provider Communication $(n=21)$, Social $(n=20)$, Feelings $(n=9)$, and Messages for Young 


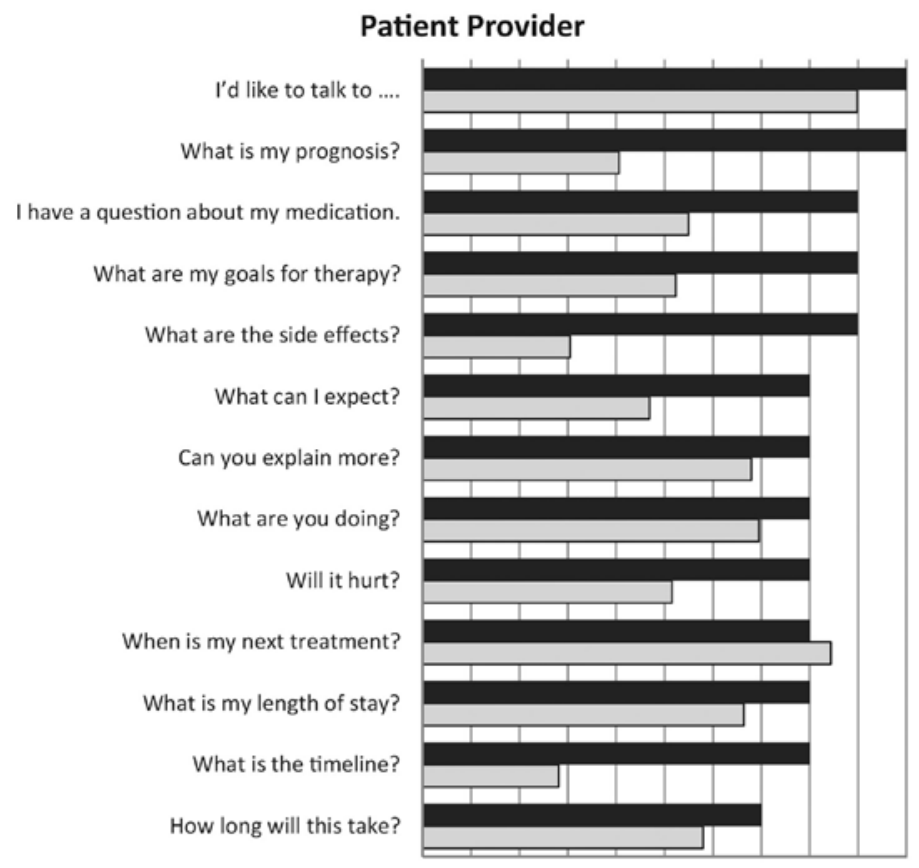

$0 \% 10 \% 20 \% 30 \% 40 \% 50 \% 60 \% 70 \% 80 \% 90 \% 100 \%$

- Participants with Disability $\quad$ Rehabilitation Care Providers

Figure 2. Percentage of Patient-Provider messages selected as important by individuals with disability and rehabilitation care providers.

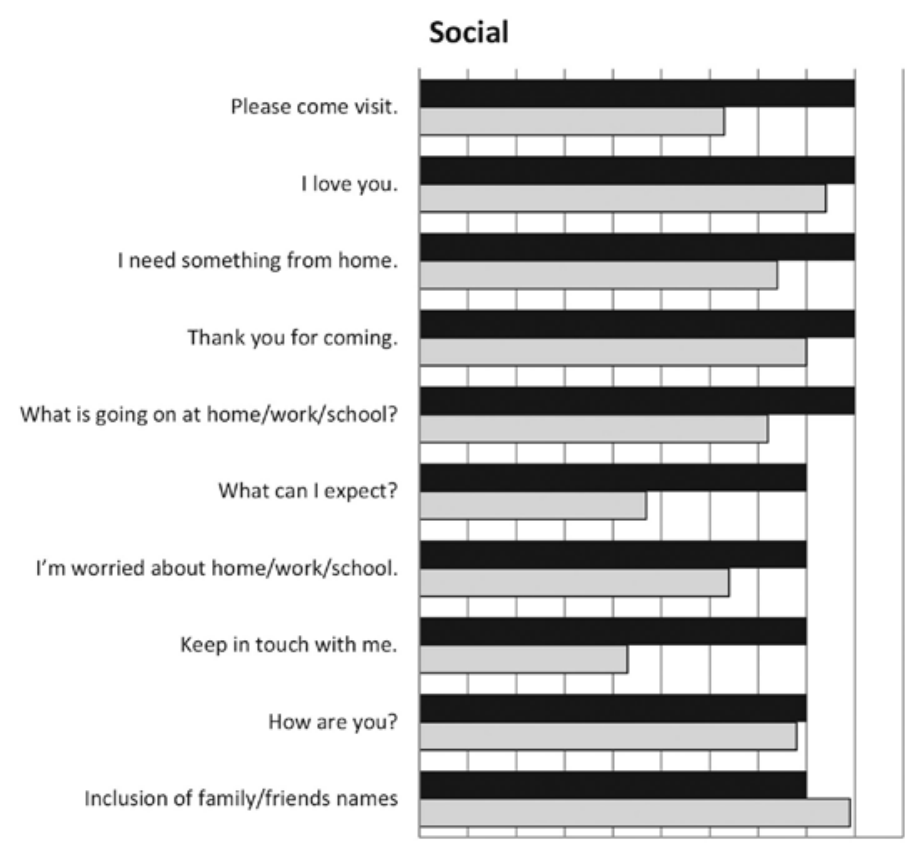

$0 \% 10 \% 20 \% 30 \%$ 40\% 50\% 60\% 70\% $80 \% 90 \% 100 \%$

- Participants with Disability $\quad$ QRehabilitation Care Providers

Figure 3. Percentage of Social messages selected as important by individuals with disability and rehabilitation care providers. 


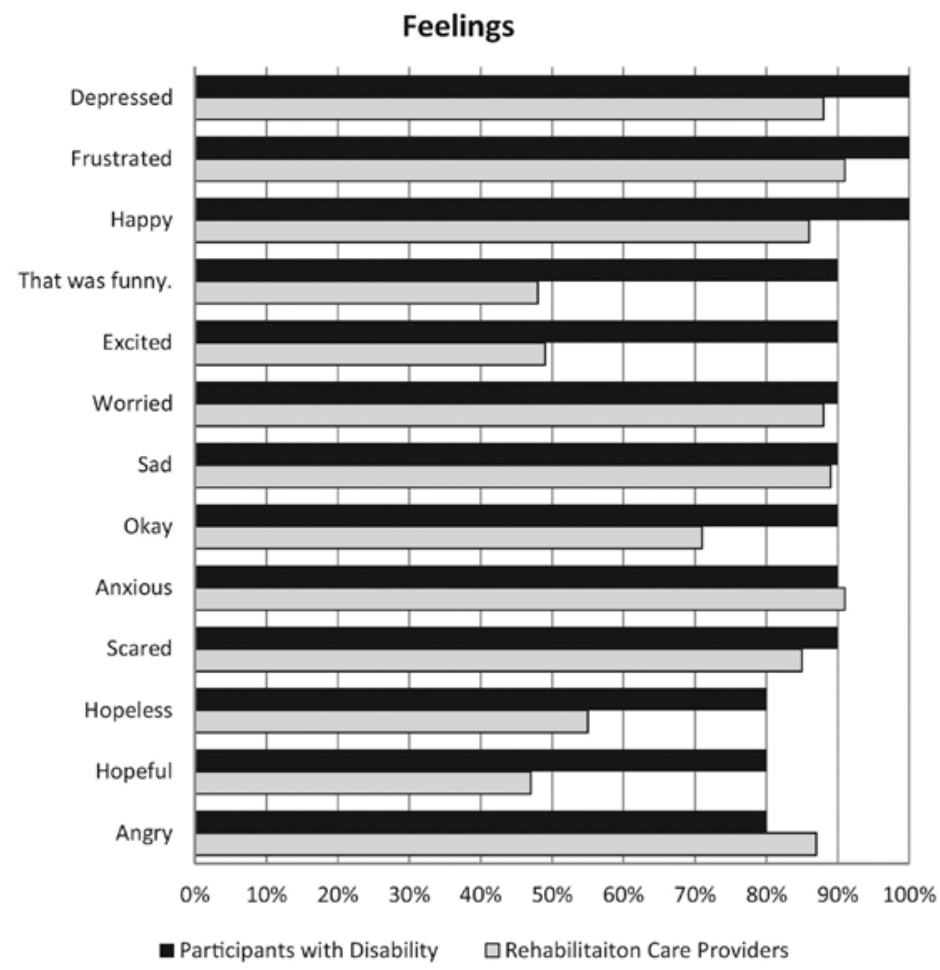

Figure 4. Percentage of Feelings messages selected as important by individuals with disability and rehabilitation care providers.

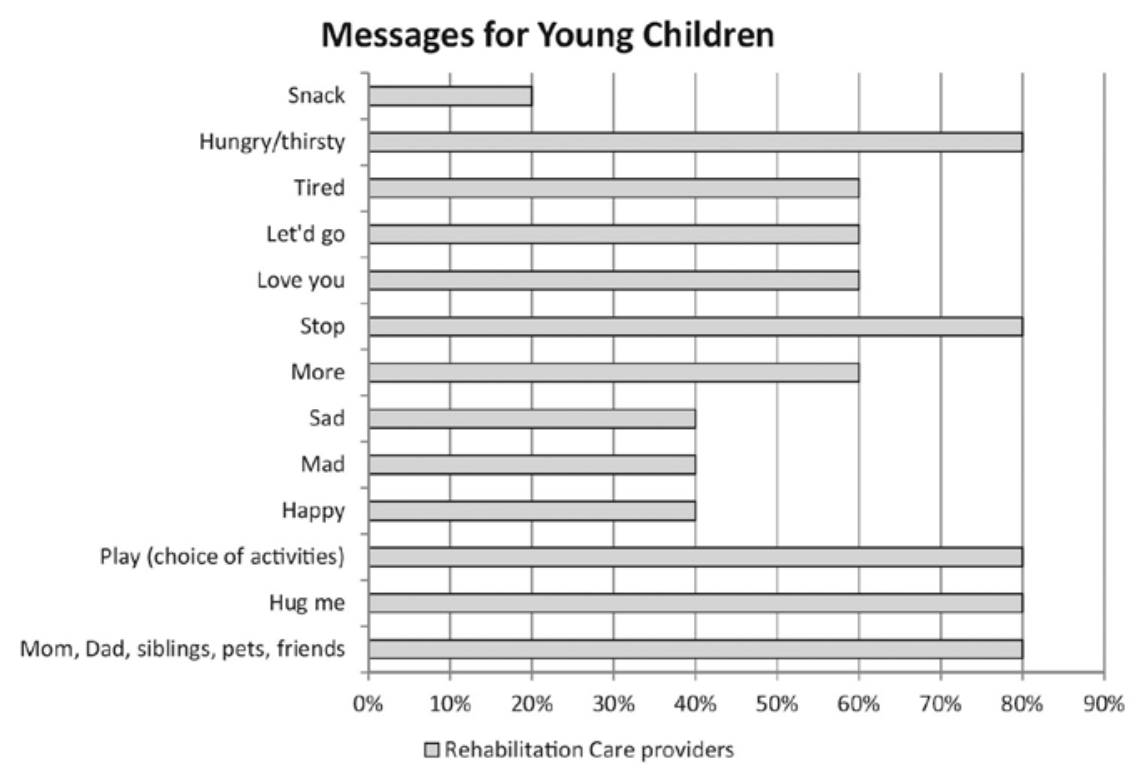

Figure 5. Percentage of Messages for Young Children selected as important by rehabilitation care providers. 
Children $(n=20)$. Themes were identified when two or more participants recommended similar messages. Under Basic Needs, the additional suggested messages included the following themes: environmental control (e.g., TV on/off, turn lights on/off; 14\% of responses), comfort (20\% of responses), bathroom needs (7\%), signs of autonomic dysreflexia ( $6 \%$ of responses), request for someone (7\% of responses), temperature control ( $7 \%$ of responses), and general itching, feelings, and medication (3\%). Under Patient-Provider Specific Communication, the additional suggested messages included the following themes: treatment plan or plan of care (29\%), pain (29\%), and requesting contact with family or care-provider (19\%). Under Social, the additional suggested messages included the following themes: requests for visits and questions about family (20\%) and gratitude (15\%). No themes emerged from the Feelings categories with only 9 suggested messages. Under Messages for Young Children, the additional suggested messages included the following themes: age-appropriate bathroom (10\%), preferred activity or toy (35\%), hurt/pain (15\%), yes/no (10\%), and activity cessation (15\%). Table 1 includes all suggested additional messages per category and theme. Messages that were suggested multiple times are only included listed in the appendix once, but an asterisk $\left(^{*}\right)$ has been placed next to the message to indicate that it was suggested multiple times.

\section{Discussion}

The capacity to communicate basic needs, health care concerns, social messages and feelings while hospitalized is critical for the wellbeing and medical safety of patients including those who are communicatively vulnerable. This study examined the perceived importance to individuals with disabilities and rehabilitation providers of messages commonly used in AAC technologies to support communication in medical settings. The results demonstrated the breadth of messages that are important for communicatively vulnerable individuals to be able to express. Equally important, the data point to the disparity that exists in the perceived importance that healthcare professional participants and participants with disabilities place on select messages. Personalization of AAC content has been identified as an important 
Table 1. Other suggested messages per category and theme (specific messages that were suggested multiple times are indicated by an asterisk).

\section{Basic needs}

Environmental control

- Need my call light

- Open/close the door

- Lights on/off

- Close/lower the window, curtain, shade.

- Fan on/off*

- Music on/off

Temperature control

- Hot/cold*

Itch

- I have an itch*

Bathroom needs

- Need to be cleaned up

- Bathroom breakdown (toilet, change, wet, BM)

- Urinal

- Bedpan

- Bladder care

- Bowel care

Request for someone

- Call family

- Nurse

- Spouse

- Call mom/dad/care provider

- Call my family to find out when they are visiting

Autonomic dysreflexia

- Headache*

- Pounding headache

Comfort

- Reposition

- Pillows

Medication request

- Medication*

Feelings

- Anxiety*

- Scared

Rest/break

- Rest

- I need a break

Other suggested messages

- Help

- Tissue

- Eat, drink, ice chips

- Mouth swab

- No or stop

- Nausea

- Yes/no

- Therapy refusal
Messages to help patients direct their cares to unfamiliar care staff

- I need a path to move my wheelchair or walker

- Pain (specific body parts)

- Short of breath

- Need RT

- Full (from eating)

- Shower

- Be gentle

- Pay attention

- Come and give me walk

\section{Patient-provider specific}

Pain messages

- When are my next pain meds scheduled?•

- Specific symptoms

- Can I have some pain medication?

- My pain medicine isn't helping

Request to contact family or care-giver

- Please contact my family.*

- Was my caregiver told this?

Treatment or plan of care

- How am I doing?

-What is the plan?

-Where am I going?

-Why do I need this?

- I don't understand.

- Why are we doing this?

Other suggested messages

- Does my insurance cover this?

- How will I pay for this?

- I can't sleep at night

- I'm anxious.

-When can I rest?

- What is your name?

\section{Social}

Requests for visits

-When will you be back to visit?*

-When are you coming?

- I'm glad to see you

Gratitude

- Thank you•

Questions about family

- How is your family?

- How are the kids?

Other suggested messages

- Turn on my TV, radio, computer, CD player.
- Help me with my cell phone, computer/tablet, remote, change channel on TV, etc.

- Get me up for (activity)

- I miss you*

- I'm sorry

- This is my friend/nurse/doctor

- Hello/goodbye

- Specific items from home

- Thank you

- Tell me what is going on with you.

- Tell me something funny that happened today

- Can you take this home for me?

\section{Feelings}

Other suggested messages

- Confused

- Curious

- Insulted

- Annoyed

- Embarrassed

- Wonderful, could be better (sarcasm)

- Irritation

- Tired

- Pain

Messages for young children

Age-appropriate bathroom

- Potty toilet*

Hurt/pain

- It hurts*

- Ouch

Preferred toy or activity

- Go outside

- Watch movie

- My blankie

- Walk

- School

- Toy (preferred options)

Activity cessation

- All done*

Communicating yes/no

- Yes/no*

Other suggested messages

- Help

- Go 
component to achieving successful transactional communication $[14,15]$. The results of this study also support the notion of personalization of AAC content to address the diverse needs/interests of varying patients.

The Basic Needs category generated the greatest number of suggested additions $(n=87)$, suggesting that current AAC strategies may notably under-emphasize the importance of these messages to effective and comprehensive patient-care provider communication. Anecdotally, participants with disability not infrequently offered a rationale for their selection while completing the survey with the researcher's assistance. For example, many participants with disability tended to select Basic Needs messages based upon their personalized needs and specific condition (e.g., "I didn't ever feel dizzy during my hospital stay"). These findings suggest that AAC designs should be simplified/ customized based on patients' unique needs to decrease the need to visually scan/ navigate extraneous content and to increase successful communication of urgent needs.

With only a few exceptions, participants with disability more frequently selected all of the messages listed under the Patient- Provider Specific, Social, and Feelings categories compared to rehabilitation care providers' responses. It is possible that while messages intended to communicate basic needs are dependent upon the specific condition of the patient, the other categories (e.g., Patient-Provider Specific, Social and Feelings) are more universal across individuals undergoing medical care. It is important for healthcare providers to understand that their patients may desire a broader range of communication options beyond communicating Basic Care needs. For example, one participant with disability indicated that the Patient-Provider Specific messages were very important to him as he wanted to know more about his acute medical condition and what was happening to him during the time he was unable to speak in the ICU. While healthcare provider perceptions might focus on the urgent care needs of the moment, it is important to recognize that many patients who are communicatively vulnerable still maintain a strong communication desire in order to direct their care, participate in care decisions, and express social closeness and feelings during this stressful time.

Balancing the breadth of messages that are important to the patient without overwhelming them and causing difficulty in finding 
messages can be challenging. This preliminary data suggests that messages related to Basic Care needs may be able to be reduced and customized based on the patient's current condition and potentially stratified into frequently used and urgent needs being offered first and less frequently used or less urgent needs offered at a secondary level. The ability personalize message options based on a patient's unique conditions needs to be quick and intuitive as the development and implementation of AAC in some medical settings often falls on the shoulders of nurses or other healthcare staff whose time and resources are limited. Having intuitive software interfaces that can offer suggestions to the end-user or the end-user's facilitator would help the process of personalization. Additionally, suggesting messages that the healthcare provider might not intuitively select (e.g., Patient- Provider Specific messages such as "what is my prognosis" or "can you explain more") may ensure that AAC technology includes options that reflect what patients need to be able to communicate in medical settings. The other messages that were suggested by the rehabilitation care providers and the participants with disabilities (Table 1) demonstrate the strong need for personalization of AAC systems.

\section{Future directions}

This preliminary study examined messages that are commonly used in AAC technologies and systems for patients in medical settings. It should be noted that the experiences of healthcare staff from a rehabilitation hospital setting were the only providers surveyed for this study. The perceptions of care providers from a variety of settings may provide additional insights into changing needs across the healthcare continuum. Additionally, the participants with disabilities were reflecting on their current (acute rehabilitation) and past experiences in medical settings (i.e., ICU, acute care hospital). Surveying participants as they are receiving care in these settings may provide differing results. Additionally, this survey targeted primarily the needs of adults with disabilities and their clinical care providers. Further work is clearly needed to define the needs of young children who are communicatively vulnerable as they relate not only to the clinical team but also to their family. Also not explored is how language, culture, and/or 
education influence the content that should be integrated within AAC. An additional and critical component to understanding how AAC messages can be developed and supported in range of medical settings would need to also include an analysis of the barriers to setting up personalized systems for individuals who require AAC. The results of this study suggest a wide range of messages with personalization are needed in order to meet the communication needs of this vulnerable population. However, we do not yet understand what barriers exist for care providers to provide customized AAC and how technology can be developed in a way to support this need by a wide range of care providers.

Disclosure No potential conflict of interest was reported by the authors.

\section{References}

[1] The Joint Commission. 2013. Sentinel Event Data: root causes by event type: 2004 to June 2013. Oakbrook Terrace (IL): The Joint Commission. Available from http://www.jointcommission.org/assets/1/18/Root Causes by Event Type_2004-202013.pdf

[2] Bartlett G, Blais R, Tamblyn R, et al. Impact of patient communication problems on the risk of preventable adverse events in acute care settings. Can Med Assoc J. 2008; 178(12):1555-1562.

[3] Blackstone S, Beukelman DR, Yorkston K. Patient-provider communication: roles for speech-language pathologists and other health care professionals. San Diego (CA): Plural Publishing Inc; 2015.

[4] The Joint Commission. 2010. Advancing effective communication, cultural competence, and patient and family centered care: a roadmap for hospitals. Oakbrook Terrace (IL): The Joint Commission.

[5] Beukelman D, Mirenda P. 2013. Augmentative \& alternative communication: supporting children \& adults with complex communication disorders. 4th ed. Baltimore (MD): Paul H. Brookes Publishing Co.

[6] Hurtig R, Nilsen ML, Happ MB, et al. 2015. Adult acute and intensive care in hospitals. In Blackstone SW, Beukelman DR, Yorkston KM, editors. Patientprovider communication: roles for speech-language pathologists and other health care professionals. San Diego (CA): Plural Publishing Inc.

[7] Hemsley B, Sigafoos J, Balandin S, et al. Nursing the patient with severe communication impairment. J Adv Nurs. 2001; 35(6):827-835. 
[8] Hemsley B, Balandin S. A metasynthesis of patient-provider communication in hospital for patients with severe communication disabilities: informing new translational research. Augment Altern Commun. 2014;30(4):329-343.

[9] Beukelman DR, Nordness AS. Patient-provider communication for people with severe dysarthria: referral policies that lead to systems change. Semin Speech Lang. 2017;38(3): 239-250.

[10] Happ MB, Tuite P, Dobbin K, et al. Communication ability, method, and content among nonspeaking nonsurviving patients treated with mechanical ventilation in the intensive care unit. Am J Crit Care. 2004;13(3):210-218.

[11] Broyles LM, Tate JA, Happ MB. Use of augmentative and alternative communication strategies by family members in the intensive care unit. Am Cri Care. 2012;21(2):e21-e32.

[12] Bardach L. Enhancing communication in hospice settings. In: Patient-provider communication: tools for speech-language pathologists and other health care professionals. San Diego (CA): Plural Publishing Inc; 2015. p. 271-301.

[13] Beukelman DR, Nordness AS. Patient-provider communication in rehabilitation settings. In: Patient-provider communication: roles for speechlanguage pathologists and other health care professionals. San Diego (CA): Plural Publishing Inc; 2015.

[14] Reichle J, Drager K, Caron J, et al. Playing the long game: considering the future of augmentative and alternative communication research and service. Semi Speech Lang. 2016;37(4):259-273.

[15] Simmons-Mackie N. Communication partner training in aphasia: reflections on communication accommodation theory. Aphasiology. 2018;32(10):12151224. 